\title{
LIFETIMES OF KEYS \\ IN CRYPTOGRAPHIC KEY MANAGEMENT SYSTEMS
}

\author{
E. Okamoto and $R$. Nakamura \\ C\&C Systems Research Raboratories \\ NEC Corporation \\ Miyamae-ku, Kawasaki 213 Japan
}

\section{INTRODDCTION}

Network architectures, such as System Network Architecture (SNA) [1], have an encryption function including key management at a functional layer. SNA uses Data Encryption standard (DES) [2] to encrypt data and keys. A data encrypting key is encrypted with a master key and transmitted before every session. However, "the lifetime of the master sey", namely, the time when the master key should be changed, is not prescribed. If the same key is used for a long time, it is probable that this secret key will be exposed.

This paper describes the lifetimes of keys. The lifetimes are the optimal key change periods, because they represent the optimal time intervals between key changes. We investigate the lifetimes of keys in two types of key distrbution schemes. One scheme is the usual scheme where the data encrypting key to be used in the next session is encrypted with an upper-level key encrypting key and transmitted to the receiving side. In the other scheme this encrypted data encrypting key is encrypted again with the data encrypting key being used at the present session and transmitted to the receiving side. In both schemes, the key encrypting key may be encrypted with more upper-level key encrypting keys. In this paper, the former scheme and the latter scheme are called SCHEME 1 and SCHEME 2, respectively. The keys Iffetime in SCHEME 2 is shown to be much longer than that in SCHEME $l$.

In the discussion, we assume that the cryptattack is based on the simplest method, namely the exhaustive key search. It may be possible to cryptanalyze in a shorter time, using statistical characteristics of encrypted data sequences, though there has 
been no such DES cryptanalysis reported so far. Hence, the lifetimes of keys described in this paper show one of the upper bounds.

Moreover, this paper deals with DES as an example. However, it utilizes only the fact that the effective key length is 56 bits, hence the discussion can be applied to other encryption algorithms.

\section{KEY DISTRIBUTION SCHEKES AND THEIR CRYPTANALYSIS METHODS}

Figure I shows two types of key distribution schemes, SCHEME 1 and SCHEME 2. In $E$ ig. $1, E$ and $D$ show the encryption transformation and the decryption transformation, respectively. $M$ shows a message and $R$ a register. The lowest level key, $K_{1}$, is a data encrypting key which is called a work key. The second level key, $k_{2}$, is used to encrypt $k_{1}$ for distribution, and so on. The highest level key, $K_{L}$, is not encrypted. It is sent via a secure channel or by a courier. Key $k_{L}$ is called a master key. Every key is generated randomiy at the sending side.

In SCHEME 2, when $\mathrm{K}_{i}$ is changed to $\mathrm{K}_{i}^{\prime}$ ', selectors SEL $_{i-1}$, SEL ${ }_{i-2}$, ..., SEL $L_{0}$ select upper 1 ines and switches $S_{i-1}$, $S W_{i-2}, \ldots, S W_{0}$ connect each decryptor output to upper 1 ines in the figure. Hence $E_{K} \quad\left(K^{\prime}{ }_{i}\right)$ is multi-encrypted with $K_{i-1}, K_{i-2}{ }^{\prime}$. , $\mathrm{K}_{1}$.

We assume that the cryptanalysis method is based on an exhaustive key search described below.

\section{Cryptanalysis method for SCHEME 1}

1) Obtain $C_{I}=E_{K_{1}}(M), \quad C_{2}=E_{K_{2}}\left(R_{I}\right), \quad--, C_{L}=E_{K_{L}}\left(K_{L-I}\right)$, where $E_{K_{i}}(M)$ shows an encrypted message $M$ with key $K_{i}$ and $C_{i}$ a cipher text.

2) Select a inaster key candidate $\mathrm{KC}_{\mathrm{L}}$.

3) Calculate lower level key encrypting key candidates $\mathrm{KC}_{\mathrm{L}-1}=\mathrm{D}_{\mathrm{KC}}\left(\mathrm{C}_{\mathrm{L}}\right), \quad \mathrm{KC} \mathrm{C}_{\mathrm{L}-2}=\mathrm{D}_{\mathrm{KC} L-1}\left(\mathrm{C}_{\mathrm{L}-1}\right), \ldots, \mathrm{KC}_{1}=\mathrm{D}_{\mathrm{KC}}\left(\mathrm{C}_{2}\right)$ and message candidate $M C=D_{K C_{1}}\left(C_{1}\right)$.

4) If $M C$ is the right message $M$, then let $K_{L}=K C_{L}$ and decode cipher texts, otherwise select another master key candidate 
and go to 3 )

Cryptanalysis method for SCHEME 2

1) Search for key $K_{1}$ from $E_{K_{1}}(M)$ and (partial) $M$. If $K_{1}$ is found, decode cipher texts until $R_{I}$ is changed.

2) Search for $k$ ey $k_{2}$ from $E_{K_{2}}\left(k_{1}\right)$ and $k_{I}$. If $k_{2}$ is found, decode cipher texts until $\mathrm{K}_{2}$ is changed.

L) Search for key $K_{L}$ from $E_{K_{L}}\left(K_{L-1}\right)$ and $R_{L-1}$. If $k_{L}$ is found, decode cipher texts until $K_{L}$ is changed.

There may be many $\mathrm{Ks}$ satisfying $\mathrm{E}_{K}(M)=C$ when message text $M$ and cipher $C$ are given. Hence, it is necessary to check $E_{K}\left(M^{\prime}\right)=C^{\prime}$ with other $M^{\prime}$ and $C^{\prime}$. If $E_{K}\left(M^{\prime}\right) \neq C^{\prime}$, continue to search for $K$. In this paper, searching for $K$ from $E_{K}(M)$ and $M$ means to find the real $k$.

If a cryptanalyst stores all cipher texts into a memory, they can all be decoded after finding the keys. However, the texts are usually quite old when the keys are cryptanalyzed, because cryptanalysis requres much time. Bence, we assume that the cryptanalyst tries to obtain online real-time messages.

Disclosure rate $\varepsilon_{L}$ is defined as

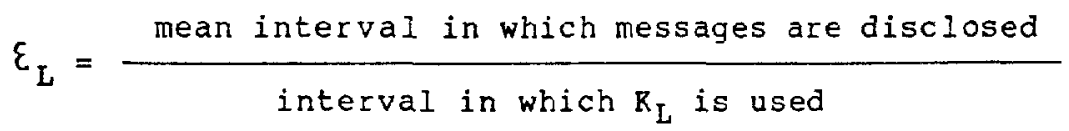

In general, rate $\varepsilon_{L}$ increases according to increase in the $k_{L}$ length.

\section{LIFETIMES OF REYS}

This section represents the lifetimes of keys as function of the number of key levels and the disclosure rate. First, the disclosure rates are derived. 
.1 Disclosure rate

(1) Disclosure rate for SCHEME I

Let $t_{L}$ be the time when $K_{L}$ is disclosed, and $T_{L}$ be the time hen $K_{L}$ is changed. Time $t_{L}$ is a random variable. Probablity ensity function $p(t)$ of disclosure of $K_{L}$ can be expressed as,

$$
P(t)= \begin{cases}\frac{1}{L A} & ;(0 \leqq t \leq L A) \\ 0 & ; \text { otherwise }\end{cases}
$$

here $A$ is the total time in which all keys need to be nvestigated. In DES case, for example,

$$
A=2^{56} \cdot \tau
$$

here $\tau$ is the time for encrypting one block.

Messages are exposed in the period from time $t_{L}$ to $T_{L^{\prime}}$ hence isclosure rate $\varepsilon_{L}$ is given by

$$
\begin{aligned}
\varepsilon_{L} & =\frac{I}{T_{L}} \int_{0}^{T_{L}}(t)\left(T_{L}-t_{L}\right) d t_{L} \\
& =\frac{C_{L}}{2 L}
\end{aligned}
$$

where $c_{L}$ is the normalized lifetime of a master key $k_{L}$, i.e.,

$$
\mathrm{c}_{\mathrm{L}}=\mathrm{T}_{\mathrm{L}} / \mathrm{A}
$$

(2) Disclosure rate for SCHEME 2

Let $t_{i}$ be the $t i$ me when the $i-t h$ level key, $k_{i}$, is exposed and let $T_{i}$ be the time when $\mathbb{R}_{i}$ is changed. Times $t_{i}$ and $T_{i}$ distributions are shown in Fig. 2. All ti are considered as random variables. 
The probability density function of disclosure of a key is

$$
p(t)=\left\{\begin{array}{l}
\frac{1}{A} ; 0 \leq t<A \\
0 ; \text { otherwise }
\end{array}\right.
$$

Disclosure rate $\varepsilon_{L}$ is given as

$$
\begin{aligned}
\varepsilon_{L} & =\frac{1}{T_{L}}\left[E \left[I\left(T_{1}-t_{I}\right)\right.\right. \\
& +E\left[I\left(T_{2}-t_{2}\right)\right]-E\left[I\left(T_{1}-t_{2}\right)\right] \\
& +E\left[I\left(T_{3}-t_{3}\right)\right]-E\left[I\left(T_{2}-t_{3}\right)\right] \\
& +\cdots \\
& \left.+E\left[I\left(T_{L}-t_{L}\right)\right]-E\left[I\left(T_{L-I}-t_{L}\right)\right]\right]
\end{aligned}
$$

where $E$ shows expectation and $I$ is the function defined as below.

$$
I(t)=\left\{\begin{array}{l}
t ; t \geq 0 \\
0 ; t<0
\end{array}\right.
$$

The minus terms in Eq. (7) come from the cases where the intervals $\left(t_{i}, T_{i}\right)$ 's are crossed each other.

$E\left[I\left(T-t_{i}\right)\right]$ is calculated as.

$E\left[I\left(T-t_{i}\right)\right]=\int_{0}^{T} p\left(t_{1}\right) d t_{1} \int_{t_{1}}^{T} p\left(t_{2}-t_{1}\right) d t_{2} \cdots \int_{t_{i-1}}^{T} P\left(t_{i}-t_{i-1}\right)\left(T-t_{i}\right) d t_{i}$ 


$$
=\frac{1}{A^{i}} \int_{0}^{\min (T, A)} d t_{1} \int_{t_{1}}^{\min \left(T, t_{1}+A\right)} d t_{2} \ldots \int_{t_{i-1}}^{\min \left(T, t_{i-1}+A\right)}\left(T-t_{i}\right) d t_{i}
$$

is $A$ is usually large, we assume that

$$
T_{i} \leq A \quad(i=1,2, \ldots, L) .
$$

Inder this condition, $E\left[I\left(T-t_{i}\right)\right]$ is given as

$$
\begin{aligned}
E\left[I\left(T-t_{i}\right)\right] & =\frac{1}{A^{i}} \int_{0}^{T} d t_{1} \int_{t_{1}}^{T} d t_{2} \ldots \int_{t_{i-1}}^{T}\left(T-t_{i}\right) d t_{i} \\
& =\frac{1}{A^{i}} \int_{0}^{T} d x_{1} \int_{0}^{x_{1}} d x_{2} \cdots \int_{0}^{x_{i-1}} x_{i} d x_{i},\left(x_{i}=T-t_{i}\right) \\
& =\frac{T^{i+1}}{(i+1) ! A^{i}}
\end{aligned}
$$

and $\varepsilon_{L}$ is given as,

$$
\varepsilon_{L}=\frac{1}{c_{L}} \sum_{i=1}^{L} \frac{c_{i}^{i+1}-c_{i-1}^{i+1}}{(i+1) !}
$$

where

$$
\begin{aligned}
& c_{i}=T_{i} / A \quad(i=1,2, \ldots L) \\
& c_{0}=0
\end{aligned}
$$

Equations (4) and (12) relate disclosure rate $\varepsilon_{L}$ to the number of key levels $L$ and the normalized keys lifetimes $c_{i}$. In SCHEME 2, it is desired that $C_{L}$ be maximum, because $K_{L}$ must be changed manually. It can be derived that $c_{L}$ is maximized at

$$
c_{1}=c_{2}=\cdots c_{L-1}=0
$$


and the maximum $c_{L}$ is given by

$$
c_{L}=\left((L+1) ! \cdot \varepsilon_{L}\right)^{1 / L} \text {, }
$$

where $\varepsilon_{L}$ is considered as a parameter (See Appendix).

The $c_{1} \cdot \cdot, c_{L-1}$ influence on $c_{L}$ will be investigated when $\mathrm{L}=2$ in detail.

Figure 3 shows the relation between $\varepsilon_{I}$ and $c_{I}$, and Figure 4 shows the effectiveness of increase of $L$ on the lifetime of key. We can see that SCHEME 2 is much stronger than SCHEME 1 for cryptanalysis.

\subsection{Lifetimes of keys for the two level key cryptosystems}

Lifetimes $c_{1}$ and $c_{2}$ in the two level key cryptosystems are investigated. These types of cryptosystems are fairly often used. From Eq.(12), the disclosure rate $\varepsilon_{2}$ for SCHEME 2 is represented as

$$
\varepsilon_{2}=\frac{c_{1}^{2}}{2 c_{2}}+\frac{c_{2}{ }^{3}-c_{1}{ }^{3}}{6 c_{2}}
$$

Figure 5 shows the relations between $c_{1}$ and $c_{2}$ for some values of a parameter $\varepsilon_{2}$.

Normalized lifetimes of keys $c_{2}$ and $c_{I}$ for SCHEME 2 are optimized by the rules below.

1) $\mathrm{c}_{2}$ is maximized.

2) $c_{1}$ is maximized under the condition that $c_{2}$ nearly equals its maximum,i.e.,

$$
c_{2}=\sqrt{6 \cdot \varepsilon_{2}}
$$

Equation (18) comes from Eq.(16). In Figure 5, the maximum points of $\mathrm{c}_{I}$ aregiven as the cross pointson the graph where $c_{2} \doteqdot \sqrt{6-\varepsilon_{2}}$. The Iine connecting these points is given by the equation, 


$$
\log c_{1}-\log 10^{-1}=3 / 2\left(\log c_{2}-\log \sqrt{6 \times 10^{-1}}\right) \text {, }
$$

namely,

$$
c_{1}=0.56 \cdot \varepsilon_{2} 0.75 \text {. }
$$

Equation (19) is given from Fig. 5 by rule of thumb.

The 1 ifetimes of master keys $\mathrm{T}_{2}$ are shown in Table 1 , when DES is employed.

For example, when $\tau=10^{-6}$ second, the master key for SCHEME 1 must be changed every year, though the master key for schemE 2 has only to be changed every 56 years to estalish $\varepsilon \leq 10^{-4}$

( 1 hour/year). When $\tau=10^{-7}$ second, SCHEME 1 must change the master key every month, whereas SCHEME 2 has only to change the master key every 5 years. Therefore SCHEME 2 is superor to SCHEME 1 on the keys lifetime.

\section{CONCLUDING REMARKS}

The keys lifetimes necessary to attain a certain low disclosure rate have been investigated for two types of schemes. DES is employed as an encryption algorithm example. This paper employs the poorest attack, namely the exhaustive attack as a cryptanalysis. There may be a more effective attack. As results, we recommend to adopt SCHEME 2 and to change the master key 'at least' within a few years.

\section{ACKNOWLEDGEMENT}

The authors wish to thank Mr. Rato, Mr. Ishiguro and Mr. Goto of NEC Corporation for helpful suggetions.

\section{REF ERENCES}

[1] Lennon, R.E., "Cryptography Architecture for Information 
Security", IBM System J., vol.17, no.2, pp.138-150, 1978

[2] Federal Information Processing Standards Publication No.46, National Burean of Standards, 1977.

[3] Okamoto,E. and Nakamura,K., "Key change periods in Cryptographic key Management systems", The proceedings of the 7-th symposium on Information Theory and Its Applications (in Japanese), pp.169-173, 1984.

\section{APP ENDIX}

The aim here is to show that the maximum of $c_{L}$ is given by Eq. (16) at $\mathrm{c}_{1}=\mathrm{c}_{2}=\ldots . \cdot=\mathrm{c}_{\mathrm{L}-1}=0$. From Eq. (12),

$$
\frac{c_{L}^{L+1}}{(L+1) !}-\varepsilon_{L} c_{L}=-\sum_{i=2}^{L} \frac{c_{i-1}^{i}}{i !}\left(1-\frac{c_{i-1}}{i+1}\right)
$$

The right-hand side of Eq.(AI) is nonnegative from Eq.(10) and Eq.(13), while the left-hand side of Eq.(Al) is nonnegative if and only if

$$
0 \leq c_{L} \leq\left((L+1) ! \cdot \varepsilon_{I}\right)^{1 / L}
$$

Hence the maximum of $c_{L}$ is $\left((L+1) ! \cdot \varepsilon_{L}\right)^{1 / L}$. When $c_{L}$ is the maximum,

$$
c_{L}=\left((L+1) ! \cdot \varepsilon_{L}\right)^{1 / L} \text {, }
$$

$c_{1}, c_{2}, \ldots, c_{L-1}$ are al 1 zero, because the right-hand side of Eq.(AI) is zero and $c_{i-1}$ does not equal $i+l$ from Eq.(10) and (13). 


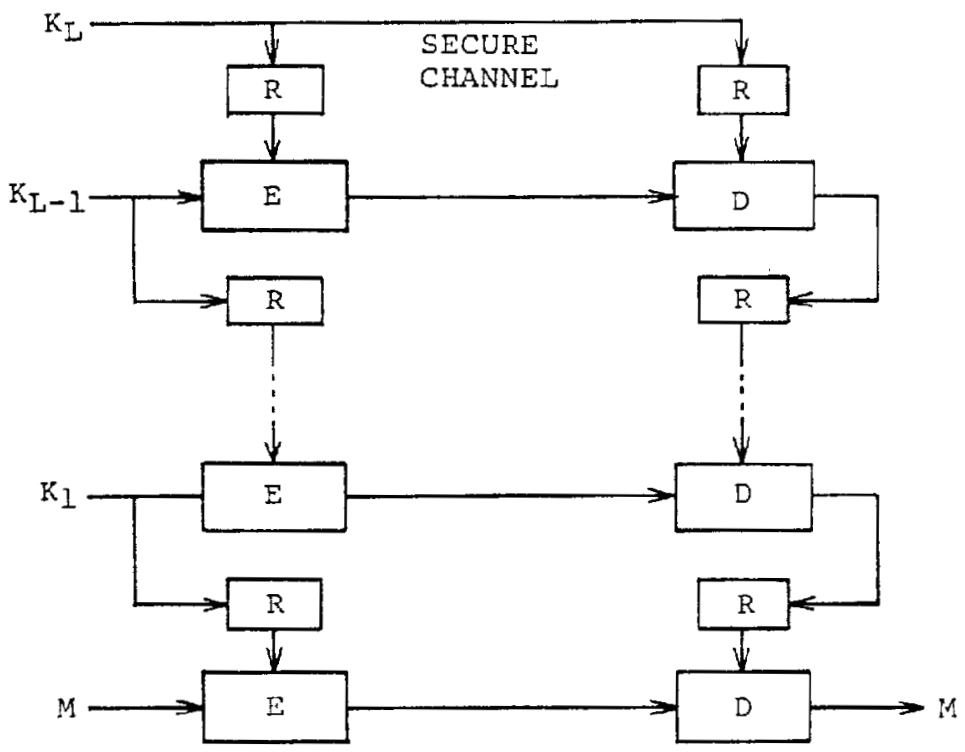

(a) SCHEME 1

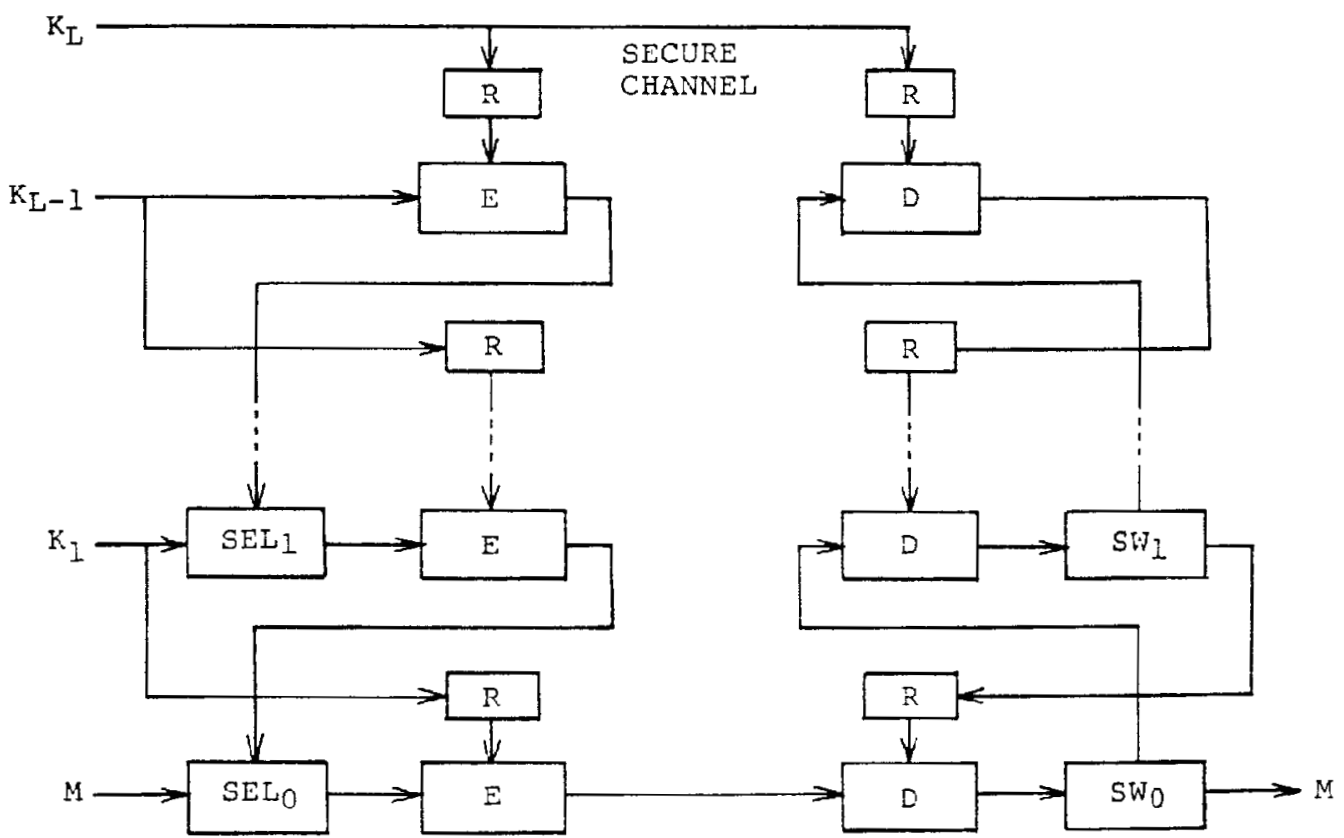

(b) SCHEME 2

Figure 1. Key distribution schemes 


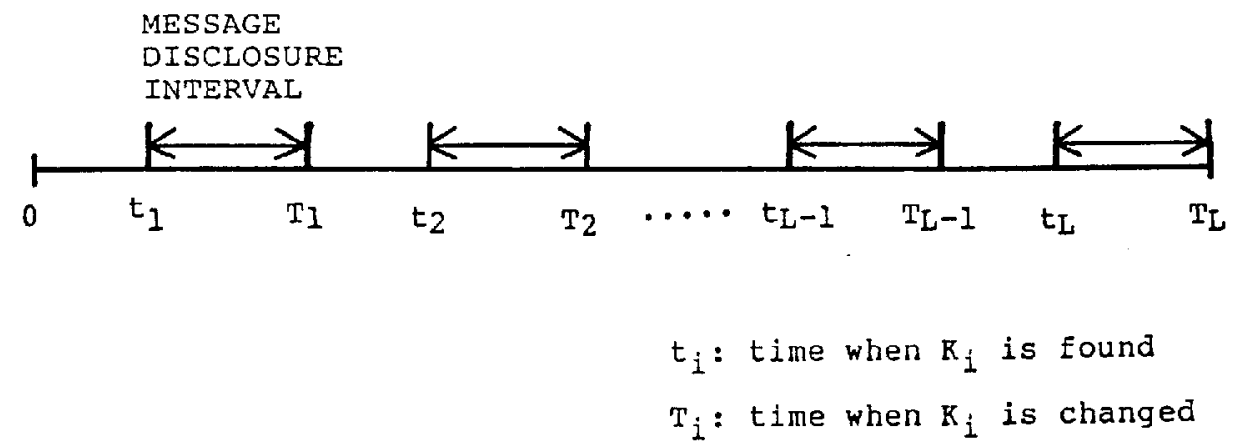

Figure 2. Times $t_{i}$ and $T_{i}$ distribution 


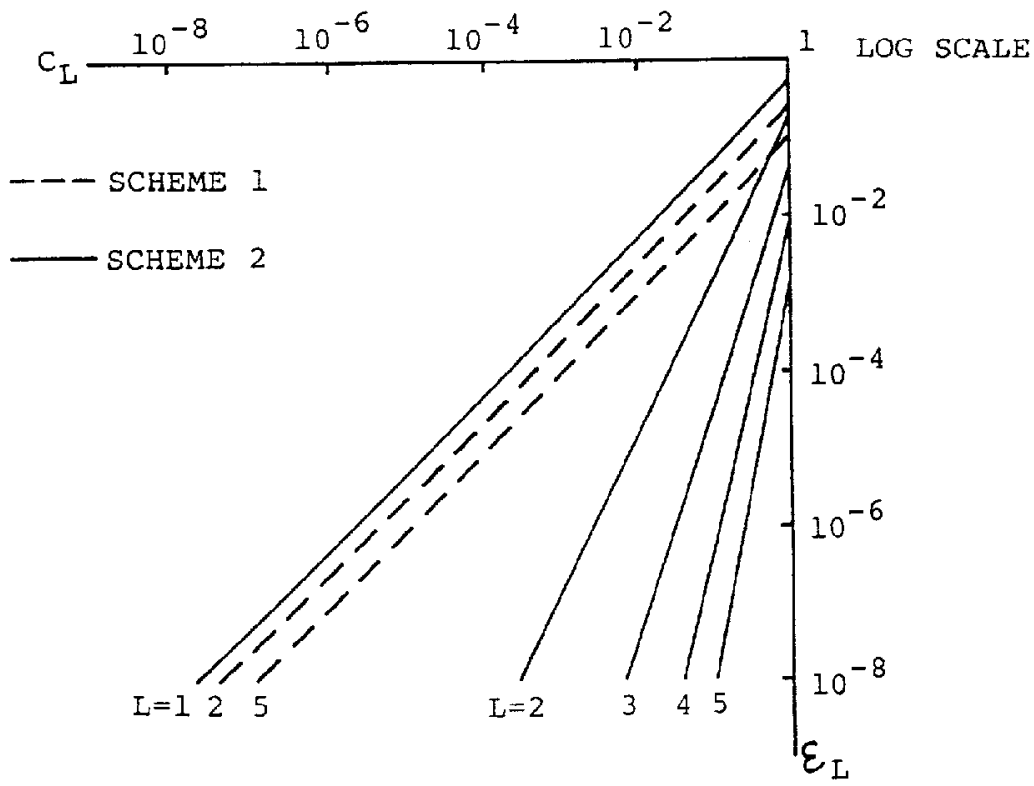

Figure 3. Relation between $C_{L}$ and $E_{I}$

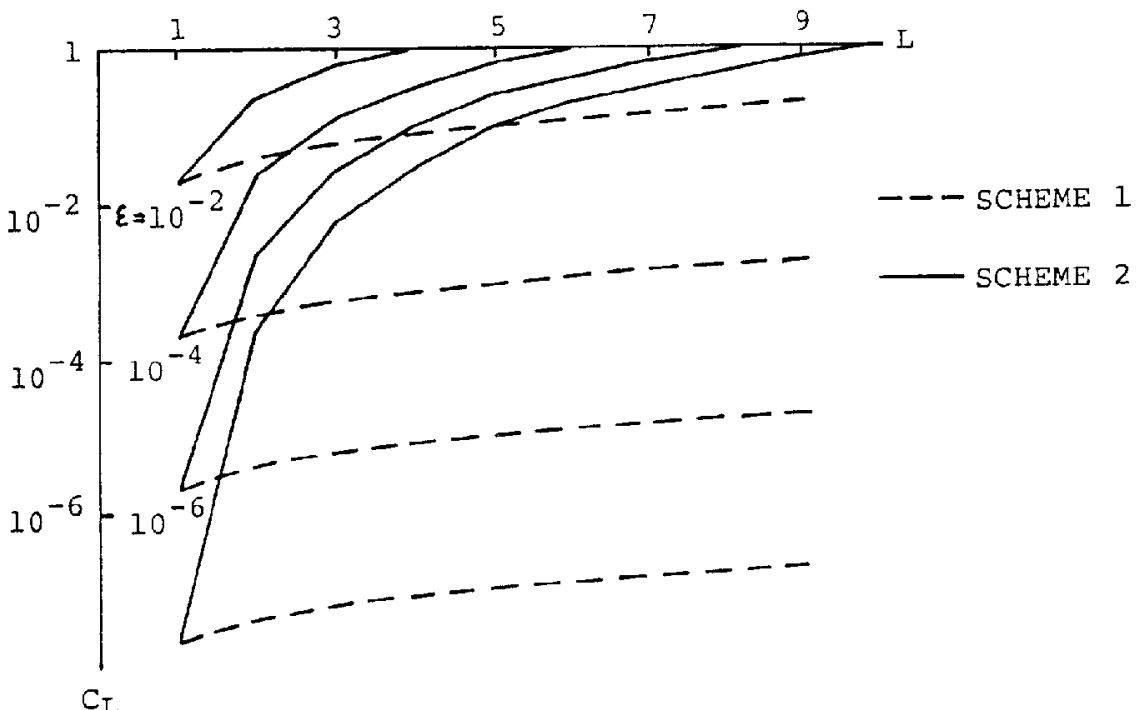

Figure 4. Relation between $C_{I}$ and $I$ 


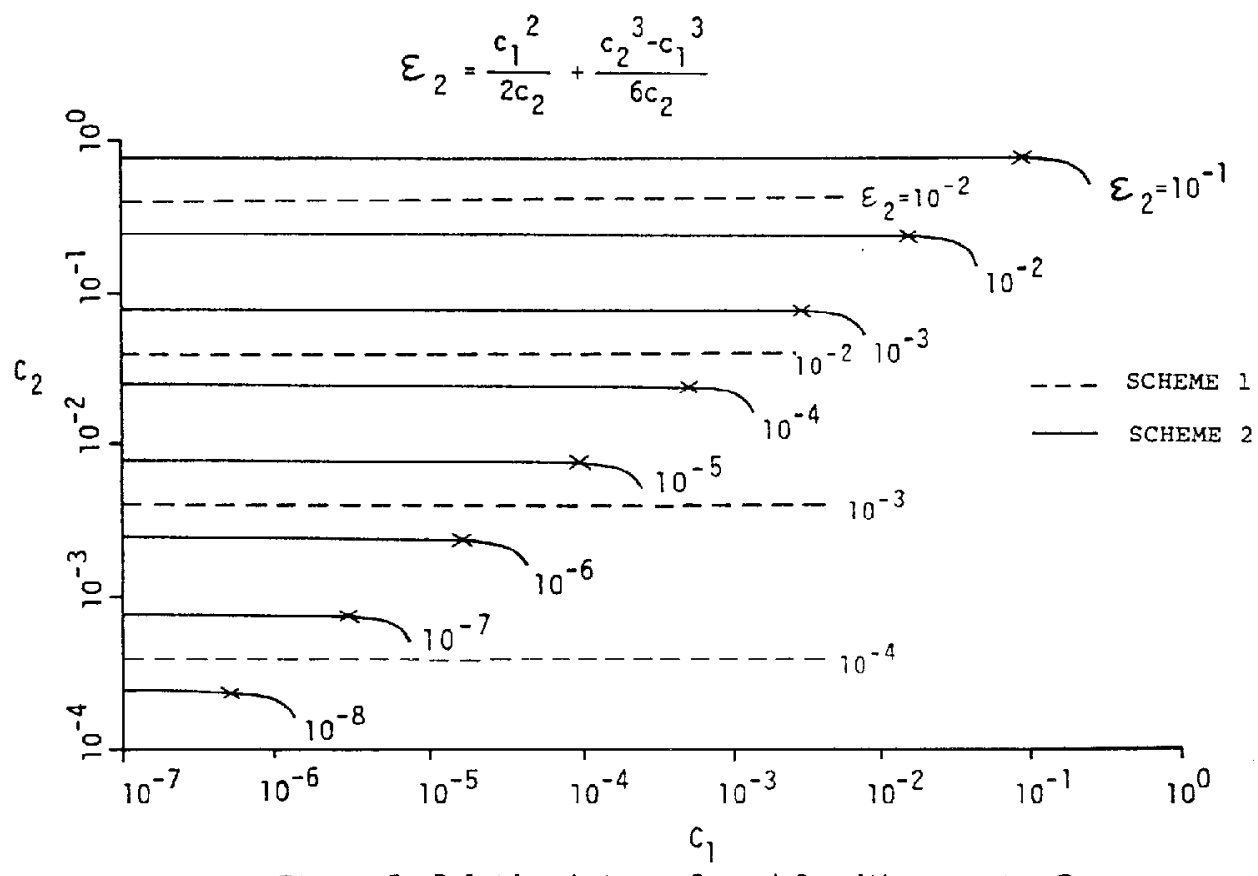

Figure 5. Relation between $C_{1}$ and $C_{2}$ with parameter $\varepsilon_{2}$ 
Table 1. Lifetimes of master keys for 2-level key cryptosystems

a) Lifetime of master key $T_{2}$ for SCHEME 1

\begin{tabular}{|c|c|c|c|}
\hline 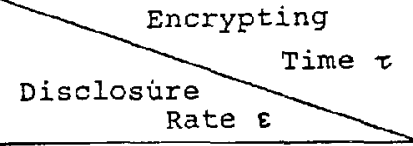 & $10^{-6}(\mathrm{~s})$ & $10^{-7}(\mathrm{~s})$ & $10^{-8}(\mathrm{~s})$ \\
\hline $10^{-1}$ & $914 \quad(y)$ & $91.4 \quad(y)$ & $9.14(y)$ \\
\hline $10^{-2}$ & 91.4 & 9.14 & 334 (d) \\
\hline $10^{-3}$ & 9.14 & $334 \quad(d)$ & 33.4 \\
\hline $10^{-4}$ & $334 \quad(d)$ & 33.4 & 3.34 \\
\hline $10^{-5}$ & 33.4 & 3.34 & $8.01(\mathrm{~h})$ \\
\hline $10^{-6}$ & 3.34 & $8.01(\mathrm{~h})$ & 48.0 \\
\hline $10^{-7}$ & $8.01(h)$ & 48.0 & $4.80 \quad(\mathrm{~m})$ \\
\hline
\end{tabular}

b) Lifetime of master key $\mathrm{T}_{2}$ for SCHEME 2

\begin{tabular}{|c|c|c|c|}
\hline Encrypting Time $\tau$ & $10^{-6}(\mathrm{~s})$ & $10^{-7}(s)$ & $10^{-8}(s)$ \\
\hline $10^{-1}$ & $1770 \quad(y)$ & $178 \quad(Y)$ & $17.8(y)$ \\
\hline $10^{-2}$ & 560 & 56 & 5.6 \\
\hline $10^{-3}$ & 178 & 17.8 & 1.78 \\
\hline $10^{-4}$ & 56 & 5.6 & 204 (d) \\
\hline $10^{-5}$ & 17.8 & 1.78 & 64 \\
\hline $10^{-5}$ & 5.5 & 204 (d) & 20.4 \\
\hline $10^{-7}$ & 1.78 & 64 & 6.4 \\
\hline $10^{-8}$ & $204 \quad$ (d) & 20 & 2 \\
\hline
\end{tabular}

